\title{
Set-shifting, central coherence and decision-making in individuals recovered from anorexia nervosa: a systematic review
}

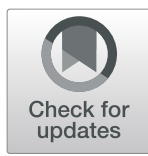

Tone Seim Fuglset巴

\begin{abstract}
Background: The aim of this study was to review the existing literature and evaluate whether deficits in setshifting, central coherence and decision-making persist in individuals recovered from anorexia nervosa (AN-REC).

Method: A systematic review approach was used. Literature was identified via searches in PubMed, PsychInfo and Embase database. The main search resulted in 158 articles. After exclusion of 135 articles, 23 articles were included in the review.

Results: The majority of studies on set-shifting showed that set-shifting difficulties persist after recovery. Central coherence might also be trait related, however findings are inconsistent. Few studies have investigated decision-making in AN-REC, however those studies that do exist suggest that decision-making is not impaired in AN-REC.

Conclusions: Novel treatment strategies based on neuroscience research are emerging, focusing on targeting the underlying mechanisms of the illness, including neuropsychological functioning. Whether these functions are trait or state related could have implications for how they are targeted in treatment.
\end{abstract}

Keywords: Anorexia nervosa, Recovered, Executive functions, Systematic review

\section{Plain English summary}

Studies have consistently shown that patients with anorexia nervosa have altered neuropsychological functions in some cognitive domains. An important question is whether these difficulties persist after the patients have recovered from their illness. If they do persist, this could be related to traits and might be considered as predisposing factors of the illness. If these difficulties normalize with recovery, they are likely a consequence of the illness state. In this study, relevant scientific literature was gathered to investigate whether deficits in the neuropsychological functions i) set-shifting, ii) global processing and iii) decision-making still persists after recovery. In total, 23 studies were included in this review article. The majority of studies on set-shifting indicate that this function is impaired in individuals recovered from anorexia nervosa.

Correspondence: Tone.Seim.Fuglset@helse-mr.no

Division of Mental Health and Addiction, Møre and Romsdal Hospital Trust, Parkvegen 84, 6412 Molde, Norway
Studies including global processing and decision-making are more unclear, as the results are inconsistent.

As anorexia nervosa is an illness that is difficult to treat, there is an urgent need for new and better treatment methods. There has been an increased interest in incorporating findings from neuroscientific research in developing new treatment strategies. These strategies are focusing on the underlying mechanisms of the illness, such as neuropsychological functioning. It would be beneficial to know whether neuropsychological deficits are related to predisposing traits or is a consequence of the illness state, as it might influence how we clinically approach the symptoms and behaviors that we see in anorexia nervosa.

\section{Background}

Anorexia nervosa (AN) is considered to be one of the most difficult psychiatric disorders to treat. There is a lack of efficient treatment approaches, especially for adult patients. The aetiology of AN is still unclear, however neuroscientific research has contributed to the 
development of theoretical models of the illness, linking the core symptoms of AN such as restrictive eating to alterations in brain circuits [1-4].

It has been suggested that neuropsychological deficits mediate between underlying neurobiological functioning and the symptom and behaviors that we see in these patients [5]. Neuropsychological studies have repeatedly demonstrated significant deficits in executive functions such as set-shifting, central coherence and decisionmaking. Set-shifting refers to the ability to move back and forward between different tasks or mental sets, and is often used as a measure of cognitive flexibility. Patients with AN often have a rigid thinking style, which also involves eating and weight. These patients also often struggle with adapting to environmental changes. Studies have consistently shown that AN patients perform worse on set-shifting tasks compared to healthy controls [6]. Central coherence refers to a bias towards processing details (local processing) at the expense of paying attention to the bigger picture (global processing). A range of studies have demonstrated weak central coherence in patients with AN, suggesting that these patients have a local processing style, and show greater global integration difficulties [7]. Furthermore, decision-making is also likely impaired in patients with $\mathrm{AN}$, as demonstrated repeatedly by several studies (rewieved in [8]). Clinical observations of patients often show that they struggle with making and trusting decisions. This could apply to decisions regarding food and what to eat, but also in other settings outside a meal situation.

A commonly raised question in this field is whether neuropsychological alterations such as deficits in setshifting, central coherence and decision-making persist after recovery. If they do persist, they could be stable traits and not related to the state of the illness. Considering an increased interest in neuroscientific-based treatment methods that has emerged during recent years, it would be beneficial to determine whether such neuropsychological deficits are trait - or state related as it could have implications for how we approach these deficits clinically.

\section{Aim of study}

The aim of the present study is to systematically review the literature to evaluate whether deficits in set-shifting, central coherence and decision-making persist in individuals recovered from AN (AN-REC), and to discuss whether these neuropsychological functions are likely to be state or trait related.

\section{Methods}

\section{Search strategy and selection of studies}

Relevant literature was identified via searches in PubMed, PSychINFO and Embase databases using the search terms [anorexia nervosa] AND [recovered OR recovery OR mental health recovery] AND [set shifting OR central coherence OR decision making].

In total, the main search resulted in 158 articles. A supplemental search was performed by a manual search in Google Scholar, which resulted in five studies. After removal of duplicates, a total of 86 articles were assessed for eligibility. Studies were included if they met the following inclusion criteria:

1. Study included an AN-REC or an AN weight recovered (AN-WR) sample and comparison with a healthy control group/normative scores.

2. Manuscript written in English.

3. Study published in peer-reviewed journals.

4. Abstracts and titles were screened for relevance and eligibility, and 57 articles were excluded. The full texts of the remaining 29 articles were examined in more detail. Of these, six studies were excluded as they were not relevant for the current review (did not include recovered patients or did not include relevant neuropsychological tests). In all, 23 articles were included in the present study. Figure 1 illustrates a PRISMA flow chart of the search strategy.

\section{Study characteristics}

One study included both males and females in their sample [9], another study did not specify the gender of the participants [10], and the remaining studies included females only. Nineteen studies included an adult sample, (age $\geq 18$ years), whereas only two studies included an adolescent sample (age $<18$ years) $[11,12]$. Two studies included both adolescents and adults [13, 14]. Definition of recovery varied across studies, see Table 1 for an overview of the different definitions of recovery.

\section{Results}

The studies included in this review were published between 2002 and 2017. The distribution across neuropsychological functions were as follows:

1) Set-shifting: 16

2) Central coherence: 8

3) Decision-making: 4

\section{Set-shifting}

A variety of tests was used to investigate set-shifting, including Fixed Set Task, Cognitive Shift Task, Trail Making Task (TMT), Brixton Test, CatBat Test, Uznadze Illusion Task, Verbal Fluency Test, Haptic Illusion Test, Wisconsin Card Sorting Test (WCST) and Berg's Card Sorting Test. The majority of studies found poorer set-shifting in ANREC compared to healthy controls $[9,10,14-16,22,24$, $26,28]$. They demonstrate that set-shifting difficulties persist after recovery, and that AN-REC does not differ from 


\section{PRISMA 2009 Flow Diagram}

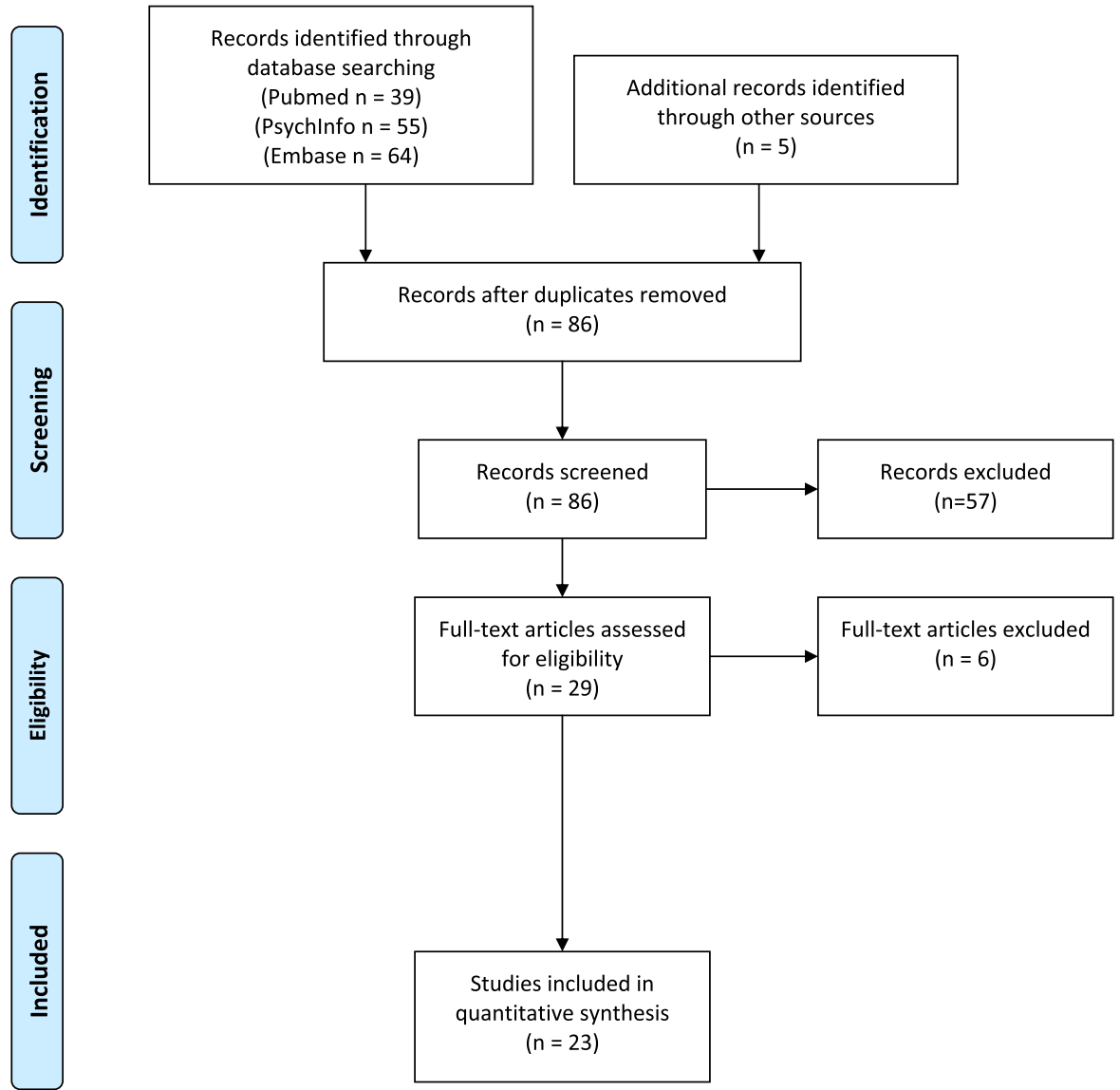

Fig. 1 PRISMA 2009 Flow Diagram

acute AN patients. Results from two of the studies showed a trend towards poorer set-shifting, but the findings were not significant [30,31]. Similarly, one study found some evidence for poorer set-shifting in AN-REC [21]. Four studies reported no differences between AN-REC and healthy controls, suggesting that there are no set-shifting difficulties in recovered patients $[11,12,18,20]$.

\section{Central coherence}

Most studies used the Rey Complex Figure Test (RCFT) to investigate central coherence, but also the Embedded Figures Test, Sentence Completion Task, Homograph Reading Task, National Adult Reading Test and Fragmented Pictures Task were utilized. Four of eight studies found no differences between the AN-REC and the acute group, suggesting weak central coherence in the AN-REC group [13, $14,19,24]$. One study found that AN-REC had no inefficiencies in global processing but a superior local processing [27]. Three studies found no significant differences in central coherence between the AN-REC group and healthy controls $[9,11,22]$.

\section{Decision-making}

Only four studies have investigated decision-making in AN-REC. In a study using intertemporal choice task, no differences were observed between AN-REC and healthy controls (HC) [29]. Similar results were found in a study using the Iowa gambling task (IGT) [17]. Another study using the IGT actually reported better performance in the AN-REC group [25]. On the other hand, the same test resulted in poorer decision-making in AN-REC Table 2 [22].

\section{Discussion}

The aim of this review was to systematically examine the literature to evaluate whether deficits in set-shifting, central coherence and decision-making persist in individuals recovered from AN-REC, and to discuss whether 
Table 1 Overview of criteria for recovery and duration of recovery. The articles are presented chronologically according to year of publication

\begin{tabular}{|c|c|c|}
\hline Authors & Criteria & Duration of recovery \\
\hline \multirow[t]{4}{*}{ Tchanturia et al., 2002 [10] } & Criteria for AN in the past & Minimum 1 year \\
\hline & BMI 19-24 & \\
\hline & Regular menstruation & \\
\hline & Normal eating patterns & \\
\hline \multirow[t]{3}{*}{ Tchanturia et al., 2004 [15] } & Stable BMI & Minimum 1 year \\
\hline & Regular periods & \\
\hline & No psychotropic medication & \\
\hline \multirow[t]{2}{*}{ Holliday et al., 2005 [16] } & Normal weight & At least 1 year \\
\hline & Regular menses & \\
\hline \multirow[t]{2}{*}{ Tchanturia et al., 2007 [17] } & BMI between 20 and 25 & At least 1 year \\
\hline & Regular menstruation & \\
\hline \multirow[t]{5}{*}{ Nakazato et al., 2009 [18] } & History of AN diagnosis according to the DSM-IV & At least 1 year \\
\hline & BMI between $18.5-24$ & \\
\hline & Regular menstrual cycles & \\
\hline & Binge and purge behavior absent & \\
\hline & No prescribed psychotropic medication & \\
\hline \multirow[t]{2}{*}{ Lopez et al., 2009 [19] } & BMI between 19 and 26 & In the current year \\
\hline & No binging, purging, food restriction or excessive exercise & \\
\hline \multirow[t]{5}{*}{ Nakazato et al., 2010 [20] } & History of AN diagnosis according to the DSM-IV & At least 1 year \\
\hline & BMI between 18.5-24 & \\
\hline & Regular menstrual cycles & \\
\hline & Binge and purge behavior absent & \\
\hline & No prescribed psychotropic medication & \\
\hline \multirow[t]{3}{*}{ Roberts et al., 2010 [21] } & Healthy BMI (> 17.5) & 1 year \\
\hline & Regular periods & \\
\hline & No AN or BN behaviors & \\
\hline \multirow[t]{3}{*}{ Tenconi et al., 2010 [14] } & Normal weight & At least 3 years \\
\hline & Regular menses & \\
\hline & $\begin{array}{l}\text { No ED symptoms and good social and } \\
\text { interpersonal outcome }\end{array}$ & \\
\hline \multirow[t]{3}{*}{ Harrison et al., 2011 [13] } & Restored regular menstruation & At least 1 year \\
\hline & No scores above 4 on EDE-Q & \\
\hline & $\mathrm{BMI}>18.5$ & \\
\hline Bühren et al., 2012 [12] & $\begin{array}{l}\text { Patients were tested before and after } \\
\text { weight rehabilitation }\end{array}$ & $\begin{array}{l}\text { Mean duration of hospital treatment was } \\
122+/-33 \text { days, range: } 57-193 \text { days }\end{array}$ \\
\hline \multirow[t]{2}{*}{ Danner et al., 2012 [22] } & $\mathrm{BMI}>18.5$ and recovered menstrual cycle & At least 12 consecutive months \\
\hline & EDE-Q and BDI not different from $\mathrm{HC}$ & \\
\hline Favaro et al., 2012 [23] & Asymptomatic & At least 3 months \\
\hline \multirow[t]{3}{*}{ Harrison et al., 2012 [24] } & Restored regular menstruation & At least 1 year \\
\hline & No scores above 4 on EDE-Q & \\
\hline & $\mathrm{BMI}>18.5$ & \\
\hline \multirow[t]{3}{*}{ Lindner et al., 2012 [25] } & No DSM-IV criteria & At least 1 year \\
\hline & BMI between 18.5 and 26 & \\
\hline & Regular menstrual cycles & \\
\hline
\end{tabular}


Table 1 Overview of criteria for recovery and duration of recovery. The articles are presented chronologically according to year of publication (Continued)

\begin{tabular}{|c|c|c|}
\hline Authors & Criteria & Duration of recovery \\
\hline & No ED specific cognitions & \\
\hline \multirow[t]{3}{*}{ Tchanturia et al., 2012 [26] } & $\mathrm{BMI}>18.5$ & At least 1 year \\
\hline & Restored menstruation & \\
\hline & Absence of ED behaviors & \\
\hline \multirow[t]{4}{*}{ Lindner et al., 2013 [27] } & No DSM-IV criteria & At least 1 year \\
\hline & BMI between 18.5 and 26 & \\
\hline & Regular menstrual cycles & \\
\hline & No ED specific cognitions & \\
\hline \multirow[t]{4}{*}{ Lindner et al., 2014 [28] } & No DSM-IV criteria & At least 1 year \\
\hline & BMI between 18.5 and 26 & \\
\hline & Regular menstrual cycles & \\
\hline & No ED specific cognitions & \\
\hline \multirow[t]{4}{*}{ Ritschel et al., 2015 [29] } & If $>18$ years old, $\mathrm{BMI}>18.5$ & At least 6 months \\
\hline & If $<18$ years old, BMI $>10$ th $\mathrm{BMI}$ percentile & \\
\hline & Menstruation & \\
\hline & No binge, purge or restrictive eating pattern & \\
\hline \multirow[t]{4}{*}{ Talbot et al., 2015 [9] } & $\mathrm{BMI} \geq 18.5$ & Past 3 months (at minimum) \\
\hline & $\begin{array}{l}\text { No binging, purging, restricting and driven } \\
\text { or compulsive exercise }\end{array}$ & \\
\hline & $\begin{array}{l}\text { Scores on all subscales of EDE-Q within } 1 \text { SD } \\
\text { of population }\end{array}$ & \\
\hline & norms & \\
\hline \multirow[t]{2}{*}{ Ely et al., 2016 [30] } & $\begin{array}{l}\text { Stable weight between } 90 \text { and 120\% of ideal } \\
\text { body weight }\end{array}$ & Prior 12 months \\
\hline & Regular menstrual cycles & \\
\hline \multirow[t]{3}{*}{ Sultson et al., 2016 [31] } & $\mathrm{BMI}>18.5$ & At least 12 months \\
\hline & Recovered menstrual cycle & \\
\hline & No differences from $\mathrm{HC}$ on the $\mathrm{EDE}-\mathrm{Q}$ & \\
\hline \multirow[t]{5}{*}{ Bentz et al., 2017 [11] } & If $>16$ years old, $\mathrm{BMI}>18.5$ & At least 1 year \\
\hline & $\begin{array}{l}\text { If } 14-15 \text { years old, } B M I-\text { percentile corrected for } \\
\text { age }>25 \text { th percentile }\end{array}$ & \\
\hline & No present ED pathology & \\
\hline & Global EDE-Q score within 1 SD of non-AN mean & \\
\hline & $M R O A S \geq 9$ & \\
\hline
\end{tabular}

$A N$ anorexia nervosa, $B D I$ Beck's depression inventory, BMI body mass index, BN bulimia nervosa; DSM-IV Diagnostic and Statistical Manual of Mental Disorders, 4th Edition, $E D$ eating disorders, EDE-Q Eating disorders examination questionnaire $H C$ healthy controls, MROAS Morgan Russel Outcome Assessment Schedule, SD standard deviation

these neuropsychological functions are likely to be state or trait related. The current review used 23 studies. The majority of studies in set-shifting suggest that this function is impaired in AN-REC. The evidence base for central coherence, on the other hand, is more unclear as findings are inconsistent. Few studies have investigated decision-making in AN-REC, however most studies so far suggest that this function is not different from healthy controls.

\section{State or trait?}

The majority of findings in this review suggest that setshifting difficulties could be trait characteristics rather than a state marker. Tchanturia et al. suggest that impaired setshifting could represent a vulnerability factor for AN [10] and that some aspects of set-shifting could be a trait rather than a state marker [15]. Likewise, in the study by Holliday et al. [16] there were no differences between acute $\mathrm{AN}$ and those who had fully recovered from AN. A 


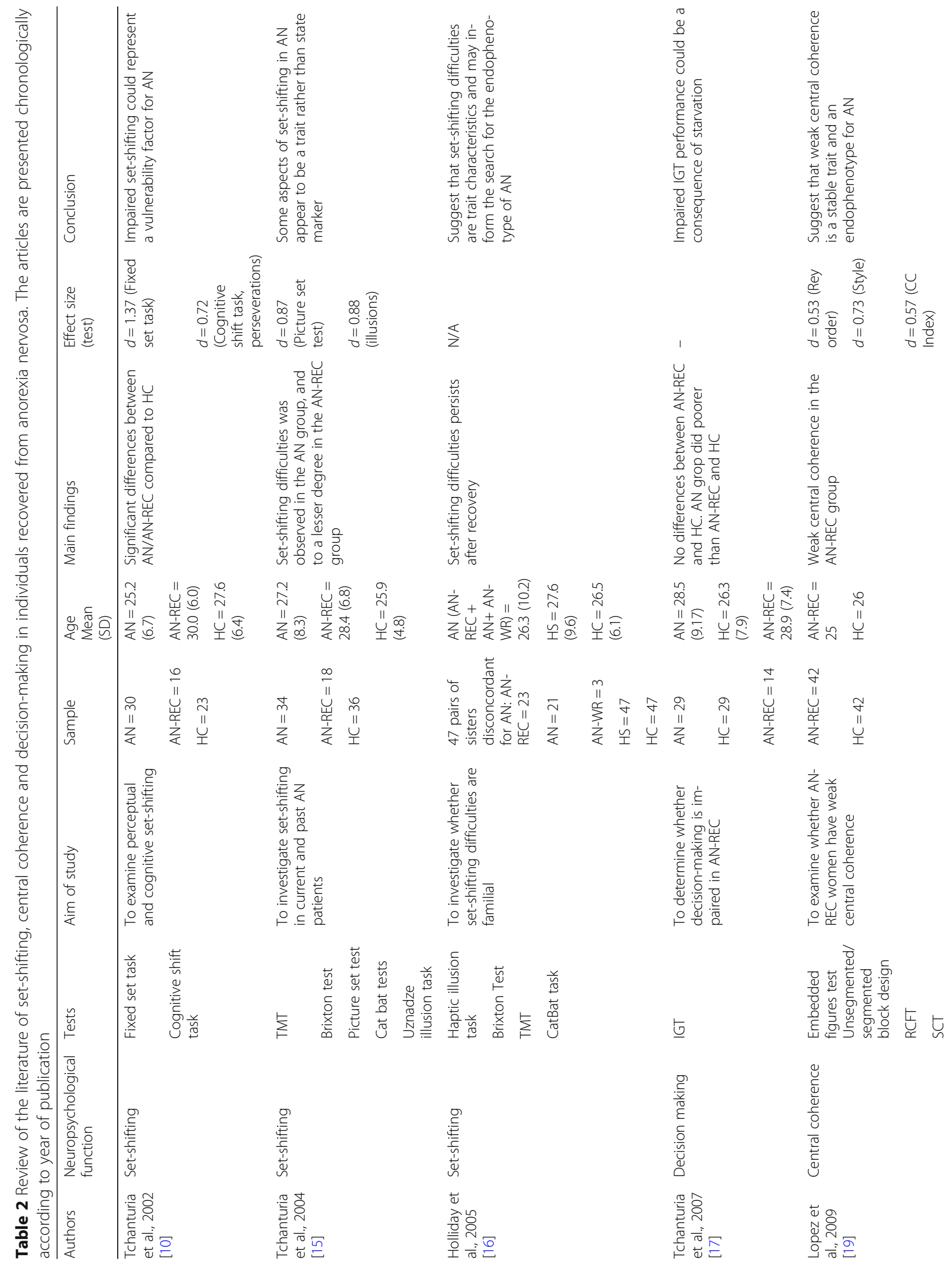




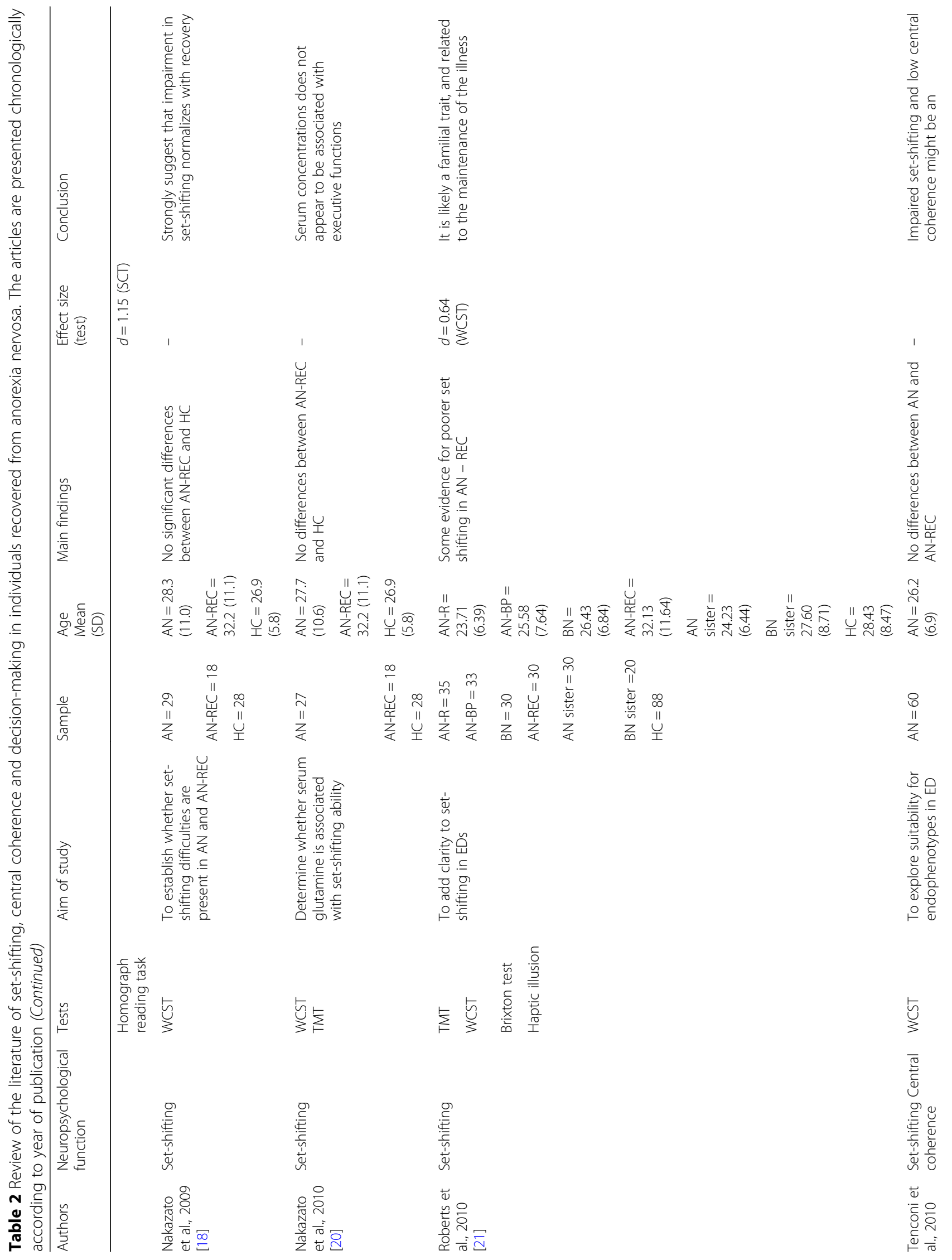




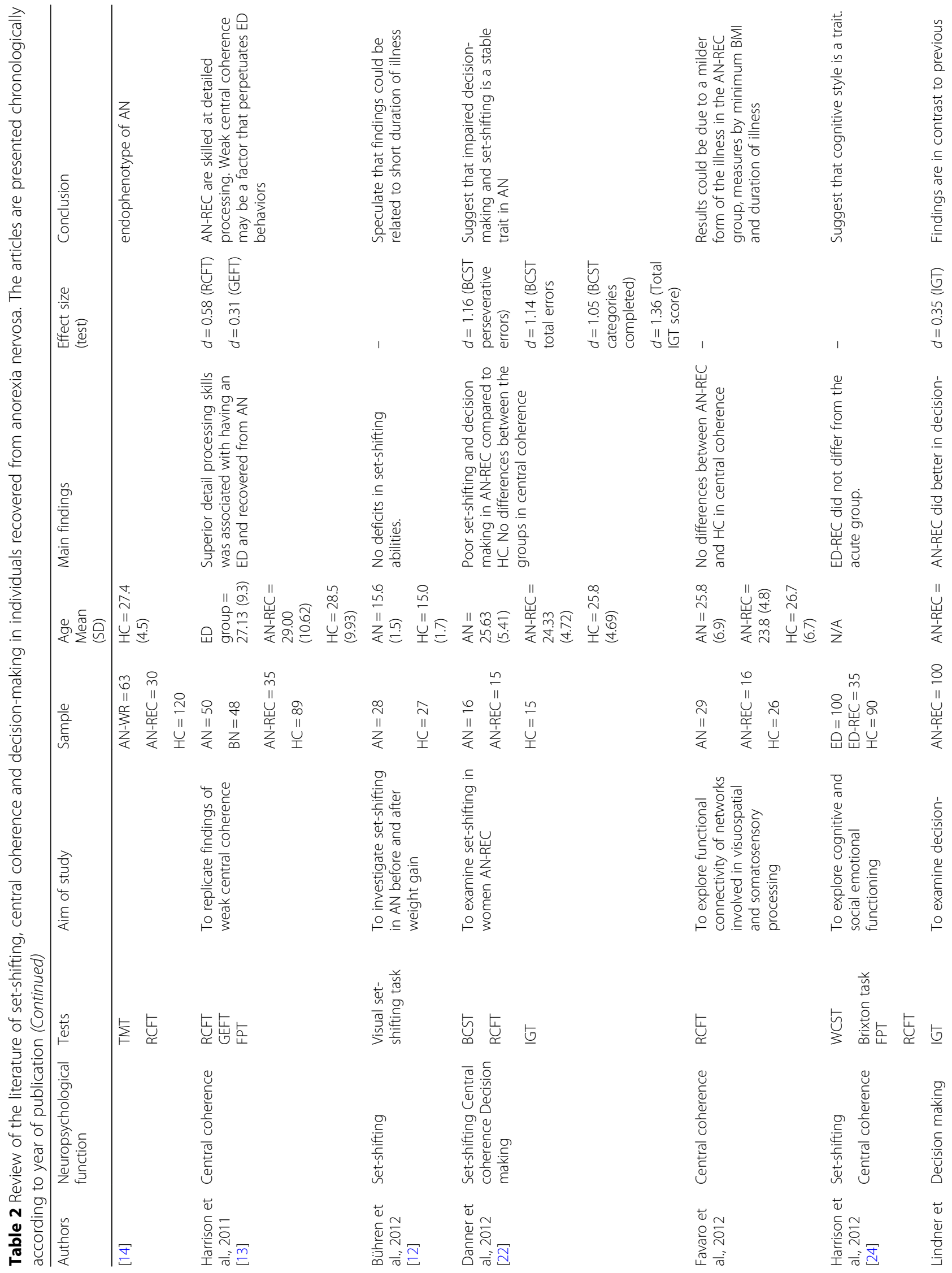




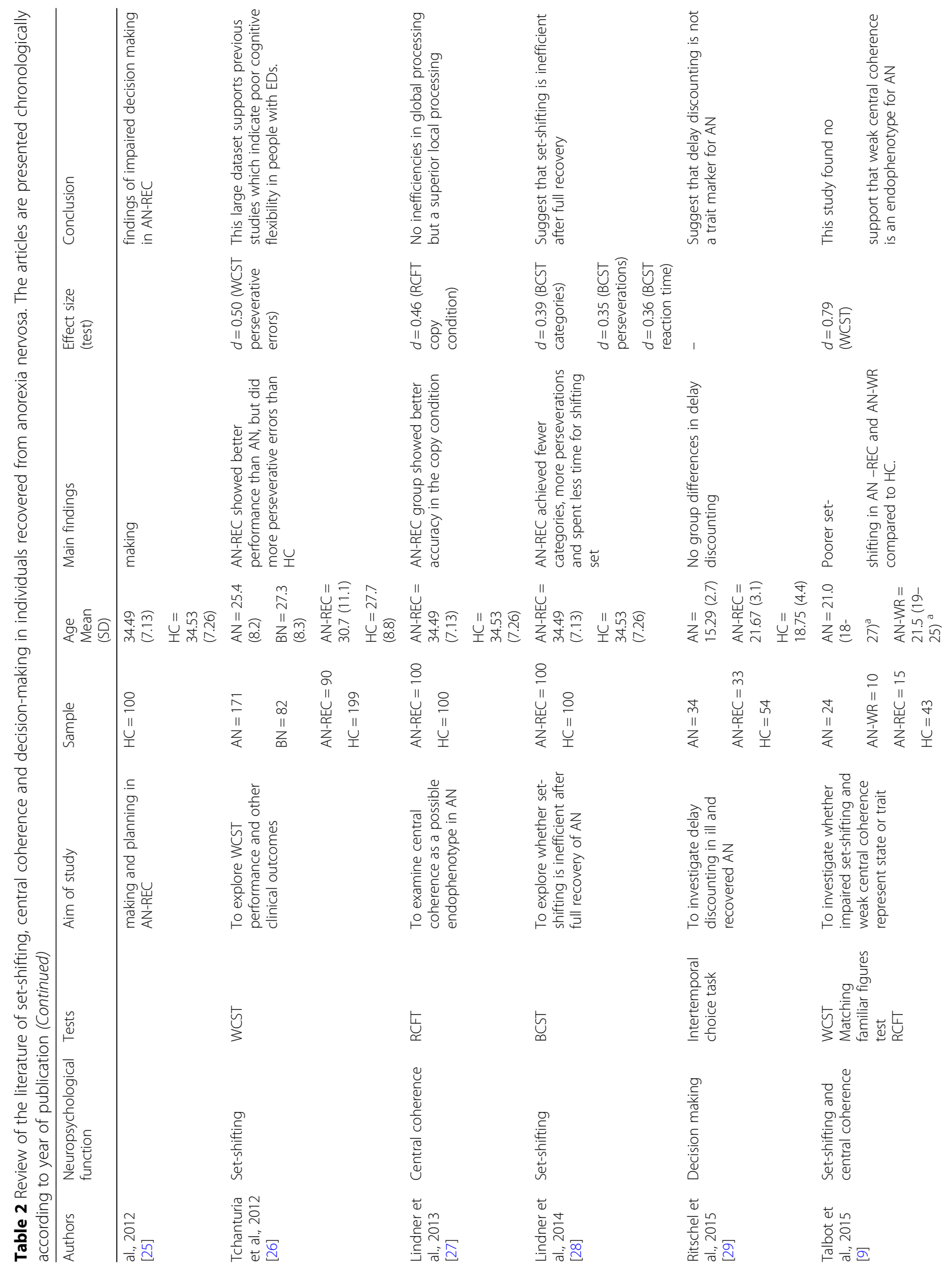


Fuglset Journal of Eating Disorders

(2019) 7:22

Page 10 of 14

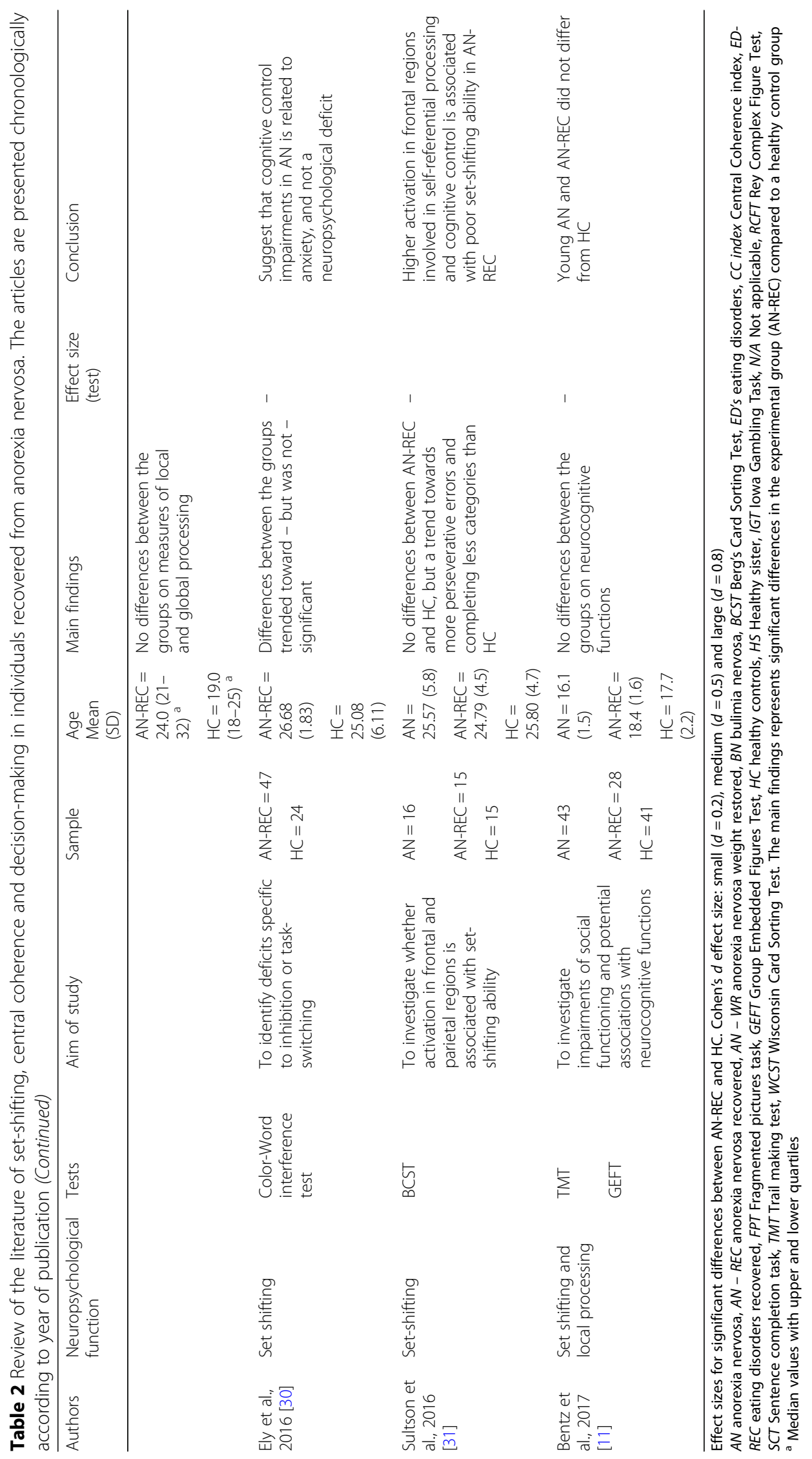


common question is whether neuropsychological deficits in AN-REC represent scarring effects after many years of starvation and underweight. Due to methodological challenges, this is difficult to determine, and still debated. However, Holliday et al. [16] further suggest that reduced cognitive and perceptual flexibility could reflect a familial trait that is associated with a greater risk of developing $\mathrm{AN}$, and not a scar due to the illness. Set-shifting difficulties as a trait is also supported by Harrison et al. [13] who reported a less adaptive cognitive style and social and emotional profile would be a trait that is associated with the eating disorder. They also suggest that this could be a maintaining factor, having the least adaptive cognitive style would be associated with a more chronic and severe form of the illness. Furthermore, they found that both individuals currently ill and recovered from AN have a fragmented perseverative cognitive style with social and emotional difficulties. A rigid cognitive style accompanied with social emotional difficulties was associated with a more persistent and severe form of illness. Danner et al. [22] also suggest that this is a stable impairment, as their results show that problems with set-shifting persist in AN-REC. Similar results was reported in the study by Talbot et al. [9], who found that individuals that have fully recovered from AN, still have impairments in setshifting. They claim that full recovery from AN does not signify normal neuropsychological functioning. The results from Lindner et al. [28] showed that AN-REC showed inefficient set-shifting or flexibility, i.e. they stayed longer to the old rule instead of changing to the new rule. In summary, these studies suggest that difficulties with set-shifting could be trait-related.

On the other hand, some studies failed to detect any differences between AN-REC and $\mathrm{HC}$ on a set-shifting task. Bentz et al. [11] did not find any differences between the groups (AN, AN-REC and $\mathrm{HC}$ ) in cognitive flexibility. Similarly, Bühren et al. [12] and Nakazato et al. [18] found no deficits in set-shifting in AN-REC. Ely et al. [30] failed to show any differences in cognitive inhibition and set-shifting in a remitted sample of AN patients and HC. They suggests that cognitive control impairments in AN is related to anxiety and does not reflect a neuropsychological deficit.

Results from studies on central coherence are more ambiguous. Based on the existing literature, it is difficult to determine whether deficits in central coherence is state or trait-related. Findings from some studies in this review show that AN-REC have persistent deficits in central coherence, while other studies indicate normal global processing in AN-REC. In the study by Danner et al. [22], those with poor set-shifting also showed weaknesses in central coherence. They suggest that these deficits could be specific to a subpopulation of individuals with $\mathrm{AN}$, and that this could be linked to a rigid thinking style. Lopez et al. [19] found that AN-REC did "extremely well" on the EFT, which benefit from enhanced detailed function and suggest that weak central coherence could be a stable trait. Likewise, Harrison et al. [13] reported that AN-REC are skilled at detailed processing and that weak central coherence may be a factor that perpetuates eating disorder behavior. They also suggest that cognitive style could be a trait of the illness [24].

Lindner et al. [27] did not find any inefficiency in global processing in the recovered group. Similarly, Talbot et al. [9] did not find any differences in measures of local and global processing. Therefore, based on the existing literature, caution should be made when determining whether central coherence is related to state or trait of AN.

Decision-making is a complex construct, and few studies have investigated decision-making in AN-REC. However, according to the existing studies, it may seem like AN-REC does not have difficulties with decision-making tasks. In a study using the IGT, Tchanturia et al. [17] found that the AN-REC group performed just as well as the $\mathrm{HC}$ group, and they question whether poor decisionmaking is related to starvation. Another study using the IGT actually reported that AN-REC did better than HC [25]. One possible explanation could be that the ANREC group have lower risk-taking behaviors. In a delay discounting task which included a monetary reward, Ritschel et al. [29] found no differences in delay discounting between AN-REC and HC. They suggest that altered self-control might be limited to disorder-relevant reinforcers, such as food. One study, using the IGT, reported that decision-making was impaired in AN-REC, and suggest that impaired decision-making is a stable trait in AN [22].

\section{Neuropsychological endophenotypes in AN?}

There has been an increased interest in determining possible endophenotypes for eating disorders. Endophenotypes can be defined as observable behavioral characteristics that underlie and contribute to disease vulnerabilities, but are not a part of the disease itself. There are three criteria of an endophenotype: i) the candidate trait is associated with the illness, ii) is state-independent, and iii) is present in unaffected family members [32]. The endophenotypes for AN are still unclear, however it has been argued that both setshifting and central coherence could be potential endophenotypes for AN [9].

In the current review, persistent impairments in setshifting is the most robust finding, as opposed to central coherence and decision-making. As an endophenotype needs to be trait-related and also present in family members, it is worth mentioning that two studies in this review also included unaffected family members. Holliday et al. 
[16] found that set-shifting deficits in women with AN were shared with their healthy sisters. Both patients and their sisters took longer to set-shift on the CatBat cognitive set-shifting task and the Haptic Illusion task. They suggest that their study provides further support for the possibility that set-shifting may be a part of the endophenotype in AN. Similarly, Tenconi et al. [14] found that AN patients and their sisters share impairments in set-shifting. They also suggest that set-shifting is a cognitive endophenotype for AN. Poor set-shifting as an endophenotype could contribute to rigid and compulsive behaviors that are often seen in individuals with AN.

Further research with unaffected family members and AN-REC is needed to further investigate endophenotypes for AN, however, set-shifting difficulties could be a possible endophenotype for this illness.

\section{Clinical implications}

There has been an increased interest in incorporating findings from neuroscientific studies to the development of treatment strategies for AN. Cognitive Remediation Therapy (CRT) involves cognitive training which addresses difficulties with flexibility and global processing [33]. The aim of this treatment is to modify and improve these deficits, and apply new cognitive skills to eatingrelated tasks. A more recently developed treatment, Temperament-based treatment with support (TBT-S) [34] is also based on neuroscientific research. Here, the patients learn strategies to cope with their deficits, and learn how to use their personality traits in constructive ways. A complete understanding of the neuropsychological deficits in patients with $\mathrm{AN}$, and whether they normalize with recovery, might influence how we develop such treatment strategies. An important issue is whether treatment should aim to alter these neuropsychological deficits, or whether the treatment should be adjusted to this specific neuropsychological thinking style.

\section{Limitations}

Some weaknesses in the literature included in this review are important to consider. First, there is a lack of consensus in the eating disorder field on a standard definition of recovery of AN. This could offer challenges when comparing results across studies. In the current review, the majority of studies include a BMI $>18.5$ and the absence of eating disorder symptoms as criteria for recovery. Although the majority of studies require that the recovered state should have been at least 1 year, other studies require a duration of 6 months and 3 months.

Conflicting results across studies could also be due to variations in clinical measures, such as duration of illness, severity degree and AN subtype (restrictive and binge/purge). Severely affected patients with a long duration of illness might have larger scarring effects compared to individuals with shorter duration of illness and less symptom severity. However, determining these variables accurately is difficult. Most studies do not define the term duration of illness and how it is assessed. In addition, duration of illness can be measured in various ways, such as by diagnosis date, what time the symptoms emerged, or what time the patient was entered into treatment. When it comes to severity degree, McGuire et al. [35] have proposed a theoretical model for the definition and conceptualization of severity of AN. According to this model, there should be a staging system, which is based on symptom severity. The main purpose of this system is to make appropriate treatment options, however it would also be beneficial for scientific purposes.

Few studies have separated patients into different subtypes. Neuropsychological difficulties could vary within the AN group, and by not separating individuals into subgroups, important findings could be overlooked. However, which group of patients that have neuropsychological difficulties is yet to be established, as studies are few and the findings are inconsistent. Lindner et al. [28] divided the AN-REC group by subtypes and found that the restricting group performed worse on a set-shifting task than HC. At the same time, Danner et al. [22] suggest that neuropsychological impairments are not a general problem for $\mathrm{AN}$, but these are specific for a subpopulation within both ill and recovered patients. Therefore, there could be subgroups of patients that have problems with set-shifting which could be independent from AN diagnostic subtype, a topic in need of further investigation.

Other differences in study design, such as sample size, might also affect the results. Lack of power calculations estimating the sample size necessary to detect significant differences between groups, could contribute to conflicting findings. In the current review, studies within the central coherence domain showed various findings. One study that failed to find significant results had a sample size of fifteen in the AN-REC group. In addition, conflicting findings could also be reflected by the large variety of tests that have been used within the same cognitive domain.

An important area of research is focused upon which factors that distinguish adolescent patients from adults. Adolescents seem to have a better prognosis than adults [36]. Studies have consistently shown that adults with AN have altered executive functioning, and some of these functions might be trait related. However, when do state difficulties occur? Moreover, are they related to the maintenance of the illness? Future studies should compare neuropsychological performance in adolescents versus adults with AN. Future studies should also focus on differentiating predisposing factors and perpetuating factors. Predisposing factors include inherent characteristic, such as genetics and personality traits, while perpetuating 
factors are conditions that maintain the symptoms of the illness. Predisposing and perpetuating factors are not necessarily the same, and determining these could contribute to the development of better treatment strategies.

\section{Conclusion}

The majority of studies in this review suggest that deficits in set-shifting persist after recovery, and could be trait related. Set-shifting difficulties could also be an endophenotype for AN as it is present in unaffected family members. Central coherence might also be trait related, however findings are inconsistent. Few studies have investigated decision-making in AN-REC, however existing studies suggest that decision-making is not impaired in AN-REC.

There is an urgent need for better treatment approaches for individuals with AN, and especially for adult patients. Novel treatment strategies based on neuroscience research are emerging, focusing on targeting the underlying mechanisms of the illness, including neuropsychological functioning. These new treatments include elements such as practicing and improving specific cognitive skills and teaching effective tools and strategies to manage eating disorder symptoms in a collaborative manner. The factors that sustain the illness are most likely different from the factors that contribute to the onset of the illness. It would therefore be beneficial to determining these factors so they could be correctly targeted in treatment.

\section{Abbreviations \\ AN: Anorexia nervosa; AN-REC: Anorexia nervosa recovered; BN: Bulimia nervosa; CRT: Cognitive remediation therapy; HC: Healthy controls; IGT: lowa gambling task; RCFT: Rey complex figure test; TBT-S: Temperament-based treatment with support; TMT: Trail making test.; WCST: Wisconsin Card Sorting Test; WR-AN: Weight-recovered anorexia nervosa}

\section{Acknowledgements}

The author would like to thank Dr. Bente Talseth-Palmer for valuable comments and for proof-reading the manuscript.

\section{Authors' contributions}

TSF conducted the literature search, analyzed the data and wrote the paper. All authors read and approved the final manuscript.

\section{Authors' information}

TSF has a PhD in Psychology from the University of Oslo and has previously worked at the Regional Department for Eating Disorders at Oslo University Hospital. She is currently in a postdoctoral position in Møre and Romsdal Hospital Trust located in Molde, Norway.

\section{Funding}

This study was funded by Møre and Romsdal Hospital Trust in Norway.

\section{Availability of data and materials}

Not applicable.

Ethics approval and consent to participate Not applicable.

\section{Consent for publication}

Not applicable.

\section{Competing interests}

The author declares that she has no competing interests.

Received: 7 February 2019 Accepted: 22 May 2019

Published online: 20 June 2019

\section{References}

1. Godier LR, Park RJ. Compulsivity in anorexia nervosa: a transdiagnostic concept. Front Psychol. 2014;5:778.

2. Kaye $\mathrm{WH}$, Fudge $\mathrm{J}$, et al. New insights into symptoms and neurocircuit function of anorexia nervosa. Nat Rev Neurosci. 2009;10(8):573-84.

3. O'Hara CB, Campbell IC, et al. A reward-centred model of anorexia nervosa: a focussed narrative review of the neurological and psychophysiological literature. Neurosci Biobehav Rev. 2015;52:131-52.

4. Steinglass J, Walsh BT. Habit learning and anorexia nervosa: a cognitive neuroscience hypothesis. Int J Eat Disord. 2006;39(4):267-75.

5. Frampton I, Rose M. Eating disorders and the brain. In: Lask B, BryantWaugh R, editors. Eating disorders in children and adolescents. 4th ed. Hove: Routledge; 2013.

6. Wu M, Brockmeyer $T$, et al. Set-shifting ability across the spectrum of eating disorders and in overweight and obesity: a systematic review and metaanalysis. Psychol Med. 2014;44(16):3365-85.

7. Lang K, Lopez C, et al. Central coherence in eating disorders: an updated systematic review and meta-analysis. World J Biol Psychiatry. 2014; 15(8):586-98.

8. Wu M, Brockmeyer $\mathrm{T}$, et al. Reward-related decision making in eating and weight disorders: a systematic review and meta-analysis of the evidence from neuropsychological studies. Neurosci Biobehav Rev. 2016:61:177-96.

9. Talbot A, Hay P, et al. Cognitive deficits as an endophenotype for anorexia nervosa: an accepted fact or a need for re-examination? Int J Eat Disord. 2015;48(1):15-25.

10. Tchanturia K, Morris RG, et al. An examination of perceptual and cognitive set shifting tasks in acute anorexia nervosa and following recovery. Eat Weight Disord. 2002;7(4):312-5.

11. Bentz $M$, Jepsen JRM, et al. Neurocognitive functions and social functioning in young females with recent-onset anorexia nervosa and recovered individuals. J Eat Disord. 2017:5:5.

12. Buhren $\mathrm{K}$, Mainz V, et al. Cognitive flexibility in juvenile anorexia nervosa patients before and after weight recovery. J Neural Transm (Vienna). 2012; 119(9):1047-57.

13. Harrison A, Tchanturia K, et al. Measuring state trait properties of detail processing and global integration ability in eating disorders. World J Biol Psychiatry. 2011;12(6):462-72.

14. Tenconi $E$, Santonastaso $P$, et al. Set-shifting abilities, central coherence, and handedness in anorexia nervosa patients, their unaffected siblings and healthy controls: exploring putative endophenotypes. World J Biol Psychiatry. 2010;11(6):813-23.

15. Tchanturia K, Morris RG, et al. Set shifting in anorexia nervosa: an examination before and after weight gain, in full recovery and relationship to childhood and adult OCPD traits. J Psychiatr Res. 2004;38(5):545-52.

16. Holliday J, Tchanturia $K$, et al. Is impaired set-shifting an endophenotype of anorexia nervosa? Am J Psychiatry. 2005;162(12):2269-75.

17. Tchanturia K, Liao PC, et al. An investigation of decision making in anorexia nervosa using the lowa gambling task and skin conductance measurements. J Int Neuropsychol Soc. 2007;13(4):635-41.

18. Nakazato $M$, Tchanturia $K$, et al. Brain-derived neurotrophic factor (BDNF) and set-shifting in currently ill and recovered anorexia nervosa (AN) patients. Psychol Med. 2009;39(6):1029-35.

19. Lopez C, Tchanturia K, et al. Weak central coherence in eating disorders: a step towards looking for an endophenotype of eating disorders. J Clin Exp Neuropsychol. 2009;31(1):117-25.

20. Nakazato M, Hashimoto $K$, et al. Serum glutamine, set-shifting ability and anorexia nervosa. Ann General Psychiatry. 2010;9:29.

21. Roberts ME, Tchanturia $K$, et al. Exploring the neurocognitive signature of poor set-shifting in anorexia and bulimia nervosa. J Psychiatr Res. 2010; 44(14):964-70.

22. Danner UN, Sanders N, et al. Neuropsychological weaknesses in anorexia nervosa: set-shifting, central coherence, and decision making in currently ill and recovered women. Int J Eat Disord. 2012;45(5):685-94.

23. Angela Favaro, Paolo Santonastaso, Renzo Manara, Romina Bosello, Giulia Bommarito, Elena Tenconi, and Francesco Di Salle (2012). Disruption of 
Visuospatial and Somatosensory Functional Connectivity in Anorexia Nervosa, Biological Psychiatry, 72:864-870

24. Harrison A, Tchanturia $K$, et al. Social emotional functioning and cognitive styles in eating disorders. Br J Clin Psychol. 2012;51(3):261-79.

25. Lindner $\mathrm{SE}$, Fichter $\mathrm{MM}$, et al. Decision-making and planning in full recovery of anorexia nervosa. Int J Eat Disord. 2012;45(7):866-75.

26. Tchanturia K. Poor cognitive flexibility in eating disorders: examining the evidence using the Wisconsin card sorting task, vol. 7; 2012. p. 1.

27. Lindner SE, Fichter MM, et al. Central coherence in full recovery of anorexia nervosa. Eur Eat Disord Rev. 2013;21(2):115-20.

28. Lindner SE, Fichter MM, et al. Set-shifting and its relation to clinical and personality variables in full recovery of anorexia nervosa. Eur Eat Disord Rev. 2014;22(4):252-9.

29. Ritschel F, King JA, et al. Temporal delay discounting in acutely ill and weight-recovered patients with anorexia nervosa. Psychol Med. 2015; 45(6):1229-39

30. Ely $A V$, Wierenga $C E$, et al. Anxiety impacts cognitive inhibition in remitted anorexia nervosa. Eur Eat Disord Rev. 2016;24(4):347-51.

31. Sultson $\mathrm{H}$, van Meer F, et al. Associations between neural correlates of visual stimulus processing and set-shifting in ill and recovered women with anorexia nervosa. Psychiatry Res Neuroimaging. 2016;255:35-42.

32. Gottesman II, Gould TD. The endophenotype concept in psychiatry: etymology and strategic intentions. Am J Psychiatry. 2003;160(4):636-45.

33. Leppanen J, Adamson J, et al. Impact of cognitive remediation therapy on neurocognitive processing in anorexia nervosa. Frontiers Psych. 2018;9:96.

34. Wierenga $C E$, Hill $L$, et al. The acceptability, feasibility, and possible benefits of a neurobiologically-informed 5-day multifamily treatment for adults with anorexia nervosa. Int J Eat Disord. 2018:51(8):863-9.

35. Maguire S, Le Grange D, et al. Staging anorexia nervosa: conceptualizing illness severity. Early Interv Psychiatry. 2008;2(1):3-10

36. Fisher $\mathrm{M}$. The course and outcome of eating disorders in adults and in adolescents: a review. Adolesc Med. 2003;14(1):149-58.

\section{Publisher's Note}

Springer Nature remains neutral with regard to jurisdictional claims in published maps and institutional affiliations.

Ready to submit your research? Choose BMC and benefit from:

- fast, convenient online submission

- thorough peer review by experienced researchers in your field

- rapid publication on acceptance

- support for research data, including large and complex data types

- gold Open Access which fosters wider collaboration and increased citations

- maximum visibility for your research: over $100 \mathrm{M}$ website views per year

At $\mathrm{BMC}$, research is always in progress.

Learn more biomedcentral.com/submissions 